\title{
Modelling the effectiveness of magnets in particle extraction
}
H. Cooper ${ }^{1}$
J. Denier ${ }^{2}$
A. $\mathrm{Ali}^{3}$
V. Chopovda ${ }^{4}$
A. Gulley ${ }^{5}$

(Received 19 April 2017; revised 11 September 2017)

\begin{abstract}
We develop a computational model to simulate the effective capture of metallic particles in industrial processes through the use of magnetic filtration systems, where these metallic particles are fragments resulting from the standard wear and tear on the machinery itself. This work is an explorative step in developing a full analytic model to better understand both the strengths and limits of such systems.
\end{abstract}

\section{Contents}

1 Introduction

M333

DOI:10.21914/anziamj.v57i0.12037, (C) Austral. Mathematical Soc. 2017. Published September 26, 2017, as part of the Proceedings of the 2015 Mathematics and Statistics in Industry NZ Study Group. ISSN 1445-8810. (Print two pages per sheet of paper.) Copies of this article must not be made otherwise available on the internet; instead link directly to the DOI for this article. Record comments on this article via http://journal austms.org.au/ojs/index.php/ANZIAMJ/comment/add/12037/0 
1.1 Fonterra . . . . . . . . . . . . . . . . M334

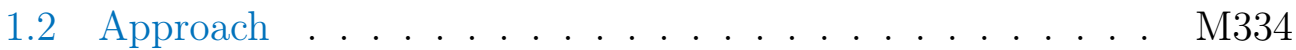

1.2.1 Data scarcity . . . . . . . . . . . . . . M336

1.2 .2 Data sensitivity . . . . . . . . . . . . . M336

1.3 The physical system . . . . . . . . . . . . . M337

1.3.1 Product . . . . . . . . . . . . . . M337

1.3.2 Metallic particles . . . . . . . . . . . . M338

1.3.3 Magnetic filtration system . . . . . . . . . . . M338

2 Fluid flow

M339

2.1 Reynolds number . . . . . . . . . . . . . . . M340

2.2 Poiseuille flow . . . . . . . . . . . . . . . . . . M341

3 Magnetic field

M342

3.1 Magnetic susceptibility . . . . . . . . . . . . . . M343

4 Total force on a particle

M344

4.1 Acceleration . . . . . . . . . . . . . . . . M344

5 Magnitude of forces

M345

6 Simulation

M346

7 Discussion

M350

8 Future steps

M351

References

M351

\section{Introduction}

This article describes work done as part of the 2015 meeting of the Mathematics in Industry Study Group of New Zealand, MINZ-2015. Specifically the 
Fonterra project workstream.

\section{$1.1 \quad$ Fonterra}

Fonterra Co-operative Group is the world's largest exporter of dairy products, manufacturing and marketing over two million tonnes of goods annually; the major part of which are powdered products, specifically milk powder. As the world leader in large-scale milk procurement, processing and management, the Fonterra Group maintains a continual focus on quality and food safety infrastructure, ensuring that they identify and remain ahead of emerging food safety risks.

One such risk is the presence of metal fragments that may be produced during the manufacturing process. Very small metallic particles occur in any industrial food processing system, naturally arising from the grinding and crushing processes, or general abrasion of metal components of the processing plant. These particles are often weakly magnetised through the same wear and tear processes prior to their erosion. Rigorous controls and standards are in place to prevent these particles affecting the final product. It is a common practice in the companies that process powdered goods to use magnetic separators as one component of a comprehensive contaminant identification and capture system.

The challenge presented at MINZ-2015 was to estimate the impact of various factors on the amount of metal attracted to magnets in a moving stream of milk powder, with a specific focus on determining if the magnets attract 'all' the metallic particles.

\subsection{Approach}

Our approach to this challenge has been to use information about the flow of the powder and structure of the magnetic filtration system, to model the path 
(and hence capture) of a metallic particle, or a group of metallic particles, through an array of magnets. By understanding the properties of the fluid flow and the properties of magnets that are in use, we determine what the magnetic field will capture, and hence against what particles the magnetic filter is effective.

As the milk powder is non-metallic, the flow is considered non-metallic. Therefore we treat the flow field and magnetic field as a decoupled system, splitting the problem into two parts:

1. an investigation of the physical properties of the milk powder flow and the flow rates within the filtration system; and

2. a study of the magnetic field generated within the filtration system and a modelling of the complex magnetic field for the specific geometry of the filtration system.

The system parameters allow the flow of powder to be treated as a steady, viscous, incompressible fluid flow. Such fluid flows are often modelled as a Stokes flow governed by a biharmonic equation [1]. However, determining appropriate boundary conditions, and a full solution, proved intractable within the time-frame of the MINZ workshop.

Instead, we use an approximation to a Poiseuille flow for the flow between two magnets in order to calculate an upper-bound on the particles' velocity within the flow. This approach supports a worst-case modelling framework that is appropriate to the industrial nature of the challenge.

MATLAB is used to simulate the effects of the magnetic field on metallic particle(s) in the de-coupled system. We further simplify the challenge by using symmetries in the filtration system and magnetic field in order to perform computations in a much smaller region of the magnetic filtration system. 


\subsubsection{Data scarcity}

There is limited data available in terms of rates and amounts of particle capture, and of the classification of metallic particles. Further, each plant may be different from other plants - in terms of design and product being produced. However, we do know details of the products, in terms of physical properties and volumes passing through the system, and of the arrangement of magnets.

Fonterra has a diverse range of factories, and a similarly diverse range of milk powder products. This diversity combined with the industrial nature of the process, seasonal timing, and general commercial sensitivity meant that data on the attraction rates of these magnetic systems was not available to the MINZ group: this lack makes any direct statistical analysis of the process impossible.

A statistical modelling approach could provide considerable depth of understanding as to the effectiveness of these filtration systems and the limits of their operation. As such, if not already part of any quality-control related process, then we recommend the development of a rigorous framework for the statistical analysis on the magnets' capture rates.

The lack of data has also partially dictated our approach-focusing on the path a metallic particle takes in a generalised flow of milk powder.

\subsubsection{Data sensitivity}

Due to the commercial nature (and hence commercial sensitivity) of this problem, we only give dimensional values sparingly. Instead, we often focus on relative orders of magnitude. 


\subsection{The physical system}

As a co-operative, Fonterra has a range of diverse factories, and diverse products - each factory is unique and there are numerous types of milk powder, each with different physical properties.

What is consistent is that milk powder is processed through a "gravity" feed, either gravity fed or free-fall through an array of magnets. The structure of the magnetic array is standardised (see subsubsection 1.3.3). The product flows through this array at $10 \%$ product by volume (90\% air).

These magnets are expected to pick up smaller metallic particles; particles greater than $3 \mathrm{~mm}$ or $2 \mathrm{~mm}$ in size are picked up by filter screens and metal detectors, respectively.

\subsubsection{Product}

As there are different types of the milk powder, the product types vary between plants. In our modelling we take the following properties of the flow that are consistent with the general nature of milk powder:

- the diameter of the milk powder particle, $\mathrm{d}_{\mathrm{s}}=2 \cdot 10^{-7} \mathrm{~cm}$;

- the bulk density of the milk powder, $\rho_{\mathrm{s}}=1.3 \mathrm{~g} \mathrm{~cm}^{-3}$;

- the density of air, $\rho_{\mathrm{a}}=0.001225 \mathrm{~g} \mathrm{~cm}^{-3}$.

Since we know the volume fractions of the milk powder and air in the flow (10\% and $90 \%$, respectively), we estimate the density of the mixture, $\rho_{\text {mix }}=0.116 \mathrm{~g} \mathrm{~cm}^{-3}$. We assume that there are a negligible amount of metallic particles in the flow.

Also, powder flows through the filtration system at rates of $2.5-25$ tons per hour. Thus, we estimate the average volume flux of the mixture, $\mathrm{Q}=$ $59865.9 \mathrm{~cm}^{3} \mathrm{~s}^{-1}$. 


\subsubsection{Metallic particles}

We are interested in milk powder processing systems. These are made using stainless steel of grades $316 \mathrm{ss}$; and $304 \mathrm{ss}$. Both are initially non-magnetic, but are made magnetic by wear. So particles that make it into the product flow will be at least weakly magnetic. For our modelling purposes we assume that particles have a radius, $\mathrm{r}$, of $1-5 \mathrm{~mm}$.

\subsubsection{Magnetic filtration system}

In the production line, milk powder flows down through a pipe and into a magnetic grating (hopper). This grating consists of one or more (usually two) layers of parallel, evenly spaced, horizontal magnetic rods, which sit perpendicular to the flow of powder.

These magnetic rods are a constructed sequence of magnetic poles and spacers, designed to maximise the strength of the local magnetic field while minimising interference between the individual rods. In this way the bar is a sequence of thin, strong alternating poles ${ }^{1}$ and wide, non-magnetic spacers.

A model based upon this configuration was developed, with each cylindrical magnet being a "programmed" series of magnets.

We focus on the situation where there are two magnet bars on the top layer, and three bars on the bottom as this captures all possible magnetic field orientations applicable to a falling particle.

${ }^{1}$ At a pole point, the entire circumference of the cylinder is positive (negative), and the next pole after the spacer is negative (positive), and so on. 


\section{Fluid flow}

The system parameters allow us to treat the flow of the mixture of milk powder, air and metallic particles as a viscous, incompressible fluid flow perpendicular to and around cylinders. The fluid flow consists mostly of air $(90 \%)$ and milk powder (10\%), metal particles have a negligible percentage and do not affect the properties of the flow.

The powder mixture flows through a pipe that is $0.15 \mathrm{~m}$ in diameter. The typical mass flow rate is $2.5-25$ tons per hour $\left(0.69-6.9 \mathrm{~kg} \mathrm{~s}^{-1}\right)$. The typical flow rate of the mixture is $0.006-0.06 \mathrm{~m}^{3} \mathrm{~s}^{-1}$ in different milk powder production plants. This information makes it possible to estimate the Reynolds number of the mixture (see Subsection 2.1).

Although a fluid flow should be modelled as a Stokes flow through the hopper and around the array of cylinders, using the biharmonic equation [1], the nature of the array and the placement of magnetic cylinders within the hopper made the determination of appropriate boundary conditions, and general calculation of the fluid flow field intractable within the time-frame of the MINZ meeting.

Instead, an approximation to Poiseuille flow through cylindrical hopper and a pair of magnets is used to calculate an upper bound on the velocity profile of magnetic particles within the flow of milk powder and air. This approach fits the risk-view of Fonterra, allowing us to focus on "worst-case" velocities, which are more relevant to applications in food safety, in lieu of a detailed model.

By taking advantage of symmetries due to the cylindrical form of magnets (see Section 3), we simplify the problem to a cross-section of the hopper, focusing on the $(x, z)$-plane. In doing this we assume that the flow disperses equally within the $(x, y)$-plane. We also assume that the $y$-axis component of a metallic particle's velocity is zero. 


\subsection{Reynolds number}

By taking into account the properties of the flow, we estimate the Reynolds number of the flow through the hopper and, in particular, between the magnets. We use the formula for the Reynolds number,

$$
\operatorname{Re}=\frac{\rho_{\text {mix }} v \mathrm{~L}}{\mu_{\text {mix }}}=\frac{v \mathrm{~L}}{v_{\text {mix }}},
$$

where:

- $\rho_{\text {mix }}$ is the density of the mixture flow;

- $v$ is the average velocity of the flow;

- $\mathrm{L}=0.0125 \mathrm{~m}$ is the characteristic length;

- $\mu_{\text {mix }}$ is the dynamic viscosity of the mixture; and

- $v_{\text {mix }}=\rho_{\text {mix }} / \mu_{\text {mix }}$ is the kinematic viscosity of the mixture.

Since the mixture that is flowing through the hopper consists mostly of the air, we estimate the kinematic viscosity of the mixture as a sum of the viscosity of air, $v_{\mathrm{a}}$, plus the change in viscosity due to the addition of the milk powder particles, $\Delta v$. Hence

$$
v_{\text {mix }}=v_{\mathrm{a}}+\Delta v, \quad \Delta v=\frac{5}{2} \frac{v_{\mathrm{a}} \mathrm{f} \rho_{\mathrm{a}}}{\rho_{\mathrm{p}}},
$$

where:

- $v_{\mathrm{a}}$ is the viscosity of the air;

- $f$ is mass concentration;

- $\rho_{\mathrm{a}}$ is the density of the air; and

- $\rho_{\mathrm{s}}$ is the density of the solid particles. 
Note that $f=m_{\mathrm{s}} / m_{\mathrm{a}}=\left(\mathrm{V}_{\mathrm{s}} \rho_{\mathrm{s}}\right) /\left(\mathrm{V}_{\mathrm{a}} \rho_{\mathrm{a}}\right)=\left(\mathrm{V}_{\mathrm{s}} / \mathrm{V}_{\mathrm{a}}\right)\left(\rho_{\mathrm{s}} / \rho_{\mathrm{a}}\right)$ and that $\mathrm{V}_{\mathrm{s}} / \mathrm{V}_{\mathrm{a}}=$ $0.1 / 0.9=1 / 9$, where $V_{s}$ and $V_{a}$ are volume fractions of milk powder particles and air, respectively. Hence

$$
\Delta v=\frac{5}{2} v_{\mathrm{a}} \cdot \frac{1}{9} \frac{\rho_{\mathrm{s}}}{\rho_{\mathrm{a}}} \frac{\rho_{\mathrm{a}}}{\rho_{\mathrm{s}}}=\frac{5}{18} v_{\mathrm{a}}, \quad v_{\text {mix }}=v_{\mathrm{a}}+\frac{5}{18} v_{\mathrm{a}}=\frac{23}{18} v_{\mathrm{a}}
$$

Having estimated the viscosity of the mixture, we estimate the Reynolds number. In our setting Re $\ll 40$, indicating that the flow is laminar, there are no vortex shedding, and our simplifications are workable.

\subsection{Poiseuille flow}

The mixture falls into the hopper from a circular opening centred above the array of magnets. The flow is centred across the gap between two magnets.

We consider the worst-case ${ }^{2}$ scenario, that the entire mixture flows through this gap. That is to say, the mixture falls out of a circular chute, of radius $r$, and then through a square in the $(x, y)$-plane of side-length $d$, where $d$ is the distance between two magnets.

We calculate the velocity profile of the Poiseuille flow between the two magnets. We assume no-slip boundary conditions for the velocity of the flow. This is workable as the boundaries are magnets and so the magnetic particles are trapped. The milk powder particles might also stick to the surface of the magnets due to the circular form of the magnets.

According to the properties of the Poiseuille flow between two magnets and the boundary conditions, the velocity of the flow has a quadratic profile. We estimate this by taking into account the total flux, $\mathrm{Q}$, in the channel between two magnets and the distance, $d$, between the two magnets:

$$
v(\mathrm{a})=0, \quad v(\mathrm{~b})=0, \quad \int_{\mathrm{a}}^{\mathrm{b}} v \mathrm{~d} \mathrm{~s}=\mathrm{Q}
$$

${ }^{2}$ Highest speed. 
where $a$ and $b$ are the boundaries of the channel between two magnets, and $\mathrm{b}-\mathrm{a}=\mathrm{d}$.

The capabilities of the plant production as well as the design of hopper set-up may differ significantly from plant to plant. In our calculations the distance $\mathrm{d}$ is $2.5 \mathrm{~cm}$ and we consider the case of a high production plant (Section 2 gives typical mass flow rates).

Our main interest for the velocity profile of the flow is to estimate the "worstcase" velocities, or the maximum velocities that a metallic particle might reach, so that it does not get trapped by the magnets when going through the filtration system.

The quadratic profile for the velocity between two magnets suggests that a particle has maximum velocity when it is equidistant from both magnets. This gives a "worst-case scenario" velocity $v_{\mathrm{p}}$ of approximately $-5 \mathrm{~m} \mathrm{~s}^{-1}$ : for all $v, v_{\mathrm{p}} \gg v$.

\section{Magnetic field}

We develop a model of the magnetic field generated by the magnetic filter, from which we simulate the force of the field on a descending particle.

While the final simulations focus on the situation where there are two magnet bars on the top layer, and three bars on the bottom, the model of the magnetic field is based around an individual magnetic pole on a single magnet bar. The model is then repeated for all poles in all magnet bars.

As each magnet is cylindrical, we model the strength of the individual magnetic field in cylindrical coordinates $(\rho, \theta, y)$. The equations associated with the 
magnetic field, $\mathbf{B}$, are

$$
\begin{aligned}
& B_{y}=\frac{-\mu_{0}}{4 \pi} M \int_{0}^{2 \pi} \int_{0}^{a}\left[\frac{R(1 / 2-y)}{\beta^{(-)}}+\frac{R(1 / 2+y)}{\beta^{(+)}}\right] d R d \Phi, \\
& B_{\rho}=\frac{-\mu_{0}}{4 \pi} M \int_{0}^{2 \pi} \int_{0}^{a}\left[\frac{R(\rho-R \cos \Phi)}{\beta^{(-)}}+\frac{R(\rho-R \cos \Phi)}{\beta^{(+)}}\right] d R d \Phi,
\end{aligned}
$$

where

- $M$ is the surface strength of the magnet,

- $\mathrm{a}$ is the radius of the magnetic pole,

- $\rho=\sqrt{x^{2}+z^{2}}$

- $\beta^{(-)}=\left[R^{2}+(1 / 2-z)^{2}+\rho^{2}-2 R \rho \cos \Phi\right]^{3 / 2}$, and

- $\beta^{(+)}=\left[R^{2}+(1 / 2+z)^{2}+\rho^{2}-2 R \rho \cos \Phi\right]^{3 / 2}$.

\subsection{Magnetic susceptibility}

The magnetic susceptibility of the particles is estimated to be $\Delta x=1.05$. This susceptibility was estimated experimentally by holding a magnet above sample magnetic particles and finding the height at which all of the magnetic partials have been attracted to the magnet. This height is an approximate lower bound on the point at which the magnetic force on the partial is equal to the gravitational force. An approximate lower bound of the magnetic susceptibility is then estimated using this information and the equation for the magnetic acceleration on a particle.

This magnetic susceptibility value is biased because all particles used in this estimation where particles that have previously been captured by a magnetic filtration system. Therefore, it is possible that they have higher then average magnetic susceptibility. 


\section{Total force on a particle}

We consider the total force exerted on the falling particle to be

$$
\mathbf{F}=\mathbf{F}_{\text {magnetism }}+\mathbf{F}_{\text {drag }}+\mathbf{F}_{\text {gravity }},
$$

where

$$
\begin{aligned}
\mathbf{F}_{\text {magnetism }} & =\frac{\nu_{\mathrm{p}} \Delta \chi}{\mu_{0}}(\mathbf{B} \cdot \nabla) \mathbf{B}, \\
\mathbf{F}_{\text {drag }} & =6 \pi \eta r\left(\mathbf{v}_{\mathrm{f}}-\mathbf{v}_{\mathrm{p}}\right), \\
\mathbf{F}_{\text {gravity }} & =\left(\rho_{\mathrm{p}}-\rho_{\mathrm{f}}\right) \mathbf{V}_{\mathrm{p}} \mathbf{g},
\end{aligned}
$$

$\mathbf{B}$ is the magnetic field, $v_{\mathrm{p}}$ is the volume of the particle, $\Delta x$ is the magnetic susceptibility of the particle, $\mu_{0}$ is a constant, $\mathbf{v}_{\mathrm{f}}$ is velocity of the fluid, $\mathbf{v}_{\mathrm{p}}$ is velocity of the particle, $V_{\mathrm{p}}$ is volume of particle, and $\rho_{\mathrm{p}}-\rho_{\mathrm{f}}$ is the relative density of the particle in the flow $\left(\rho_{\mathrm{f}}\right.$ is assumed to be negligible compared to $\rho_{\mathrm{p}}$ ).

\subsection{Acceleration}

From the equations above we derive the following components of the particles acceleration:

$$
\begin{aligned}
\mathrm{a}_{\mathrm{drag}} & =\frac{12 \pi v \mathrm{r}}{\mathrm{m}_{\mathrm{p}}}\left(\mathbf{v}_{\mathrm{f}}-\mathbf{v}_{\mathrm{p}}\right) ; \\
\mathrm{a}_{\text {magnetism }} & =\frac{\Delta \chi}{\rho_{\mathrm{p}} \mu_{0}}(\mathrm{~B} \cdot \nabla) \mathrm{B} ; \\
\mathrm{a}_{\text {gravity }} & =-9.8 z .
\end{aligned}
$$


Figure 1: Cross-section of the magnetic acceleration on a magnetic particle-a plan view through the upper two magnetic bars. White boxes indicate the magnetic dipoles that make up the magnetic bars.

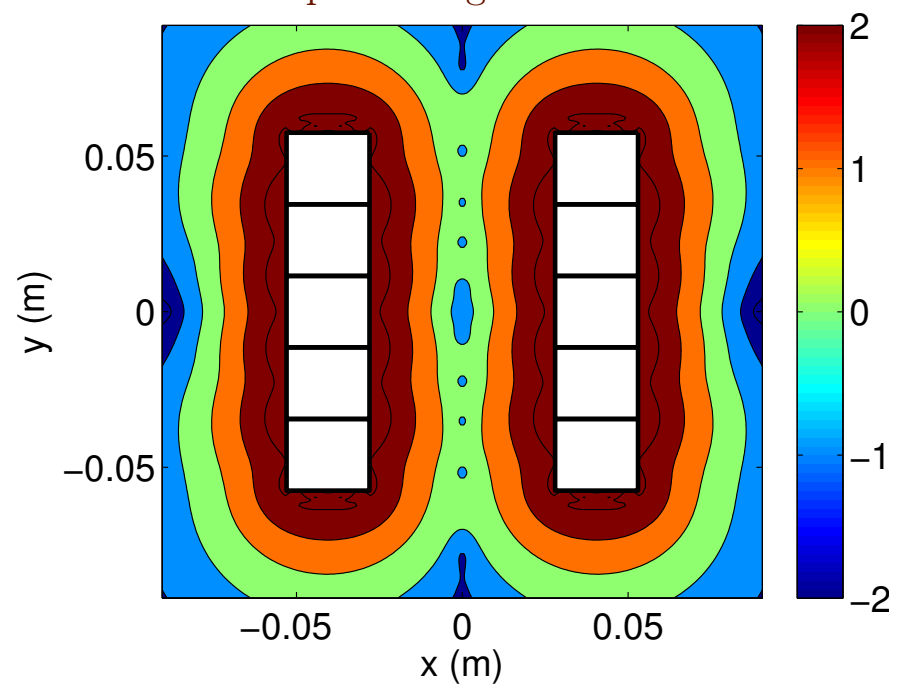

\section{$5 \quad$ Magnitude of forces}

In order to understand the most important forces on the magnetic particle we perform order of magnitude calculations. These are done assuming a spherical $1 \mathrm{~mm}$ diameter particle is stationary in a $5 \mathrm{~m} \mathrm{~s}^{-1}$ flow and is radially $25 \mathrm{~mm}$ from the edge of a single magnetic pole. The parallel magnet bars are typically $\sim 50 \mathrm{~mm}$ apart. These calculations give

$$
\begin{aligned}
\mathrm{a}_{\text {drag }} & \sim 10^{-3} \mathrm{~m} \mathrm{~s}^{-2}, \\
\mathrm{a}_{\text {magnetism }} & \sim 10^{0} \mathrm{~m} \mathrm{~s}^{-2}, \\
\mathrm{a}_{\text {gravity }} & \sim 10^{1} \mathrm{~m} \mathrm{~s}^{-2} .
\end{aligned}
$$

As $a_{\text {drag }}$ is considerably smaller than $a_{\text {magnetism }}$ and $a_{\text {gravity }}$ it is less important that the fluid flow problem is accurately computed for a particle of $1 \mathrm{~mm}$ 
Figure 2: Cross-section of the magnetic acceleration on a magnetic particle$(x, z)$ cross-sections through all five magnetic bars, though the magnetic poles.

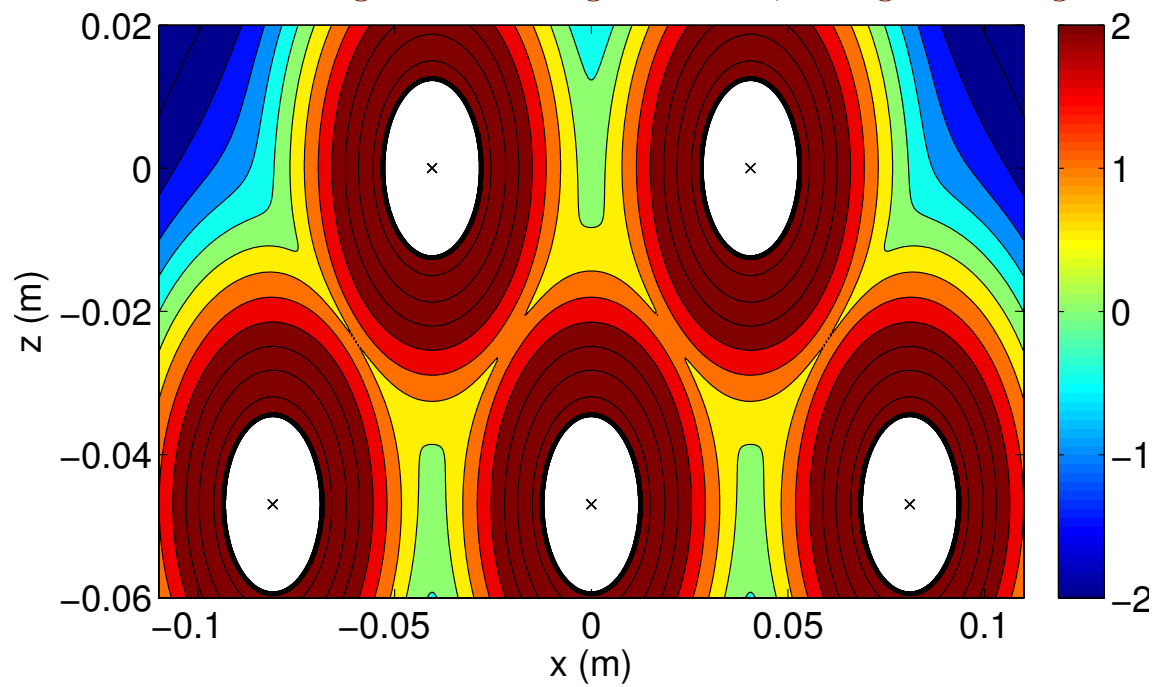

diameter. As $a_{\text {drag }} \propto r^{-2}$ the drag forces become more important for $r<$ $10^{-4} \mathrm{~m}$ and dominate for $r<10^{-6} \mathrm{~m}$. While $a_{\text {magnetism }}$ is smaller than $a_{\text {gravity }}$ it increases rapidly as the particle gets closer to the magnetic pole. Figures 1-3 show cross-sections of the magnetic acceleration on a $1 \mathrm{~mm}$ diameter particle.

\section{Simulation}

We perform numerical modelling of particle paths as they fall through the magnetic array. This is achieved through numerical integration of the acceleration on a particle using the Improved Euler's method. For the computation of $a_{\text {drag }}$ the simulations assume the fluid flow is homogenous and $1 \mathrm{~m} \mathrm{~s}^{-1}$ in the downwards direction. All particles were assumed to have an initial downwards velocity of $1 \mathrm{~m} \mathrm{~s}^{-1}$ and are spherical with a diameter of $1 \mathrm{~mm}$. The numerical model was validated qualitatively by dropping magnetised particles 
Figure 3: Cross-section of the magnetic acceleration on a magnetic particle$(x, z)$ cross-sections through all five magnetic bars, mid-way between two sets of magnetic poles.

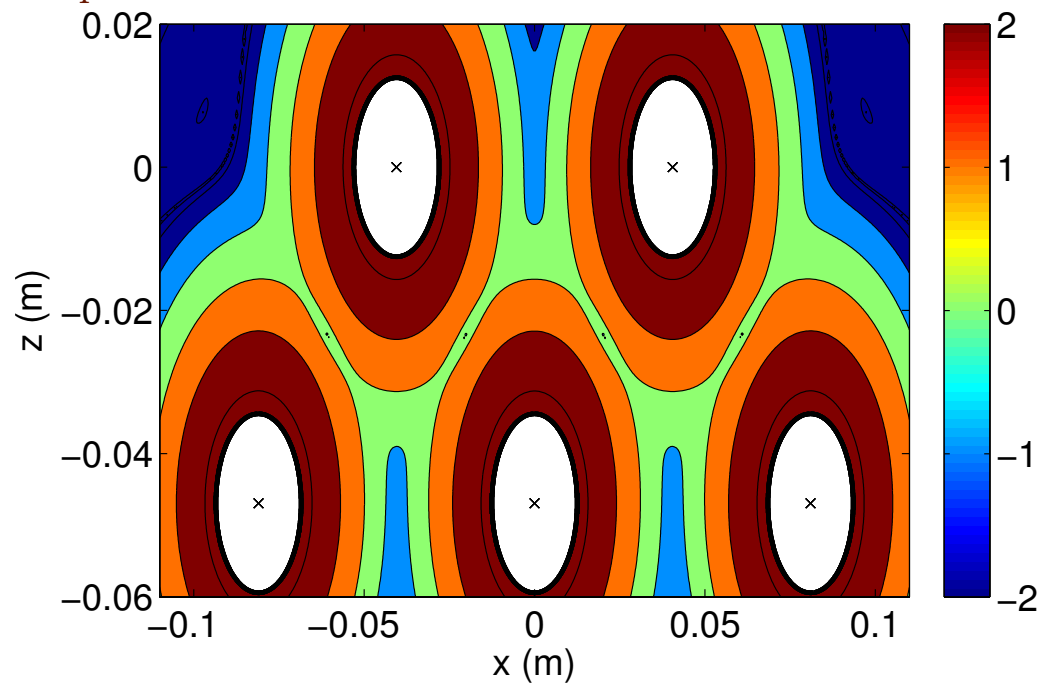

through a sample magnet array. It is also known that the magnetic force of the model is approximately correct because of the magnetic susceptibility estimation process. Therefore, these results provide qualitative estimates on how magnetic particles behave as they are falling through the magnetic bar array. Three examples of modelled trajectories, from three different starting positions are given in Figures 4-6.

Monte Carlo simulations are carried out to investigate the percent of particles captured. Two different release zones for the particles are considered: Zone 1, which is $6 \mathrm{~cm}$ high; and Zone 2, which is $40 \mathrm{~cm}$ high. We use these release zones as the particles released from Zone 2 will be traveling twice as fast through the magnets as the particles released from Zone 1. Due to the symmetry of the magnets these release zones can be confined to small rectangular areas. Figure 7 depicts these areas. 
Figure 4: Sample particle trajectories (blue) from a $40 \mathrm{~cm}$ high starting position in-line with poles.
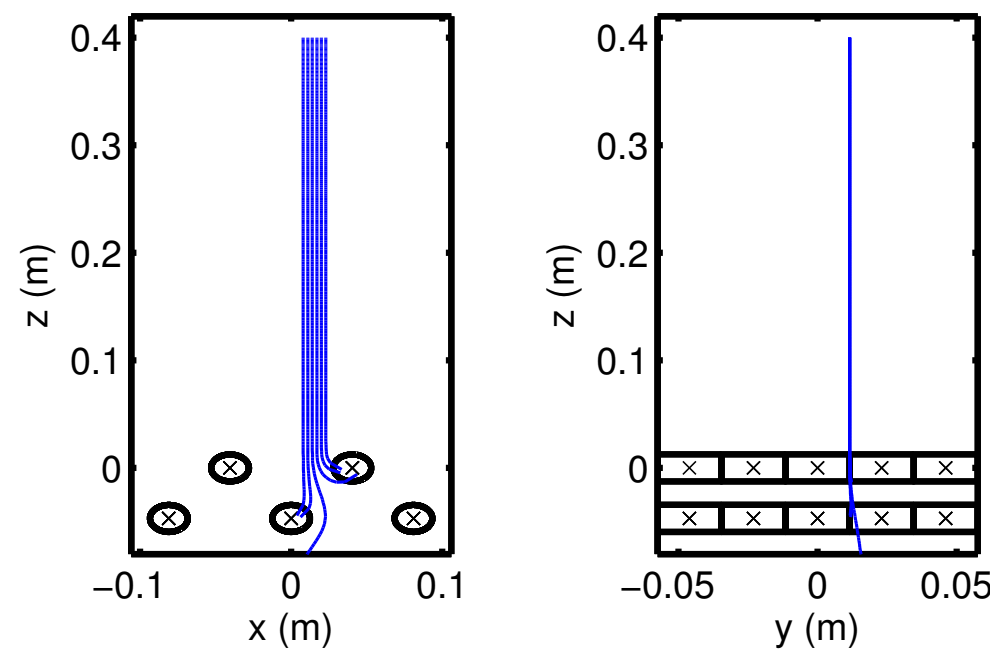

Figure 5: Sample particle trajectories (blue) from a $20 \mathrm{~cm}$ high starting position in-line with poles.
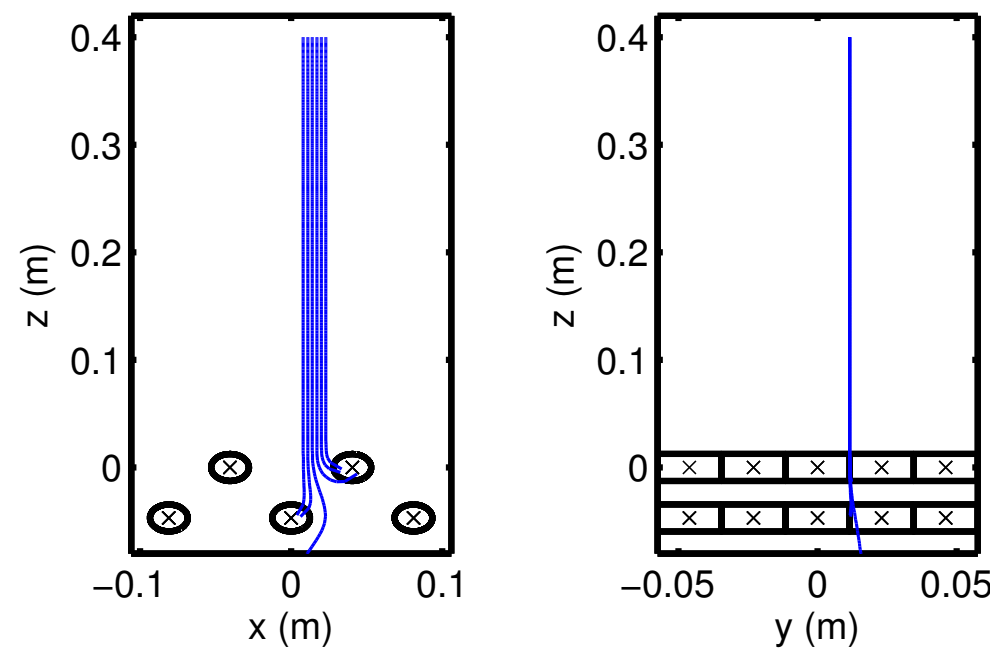
Figure 6: Sample particle trajectories (blue) from a $20 \mathrm{~cm}$ high starting position out of line with poles.
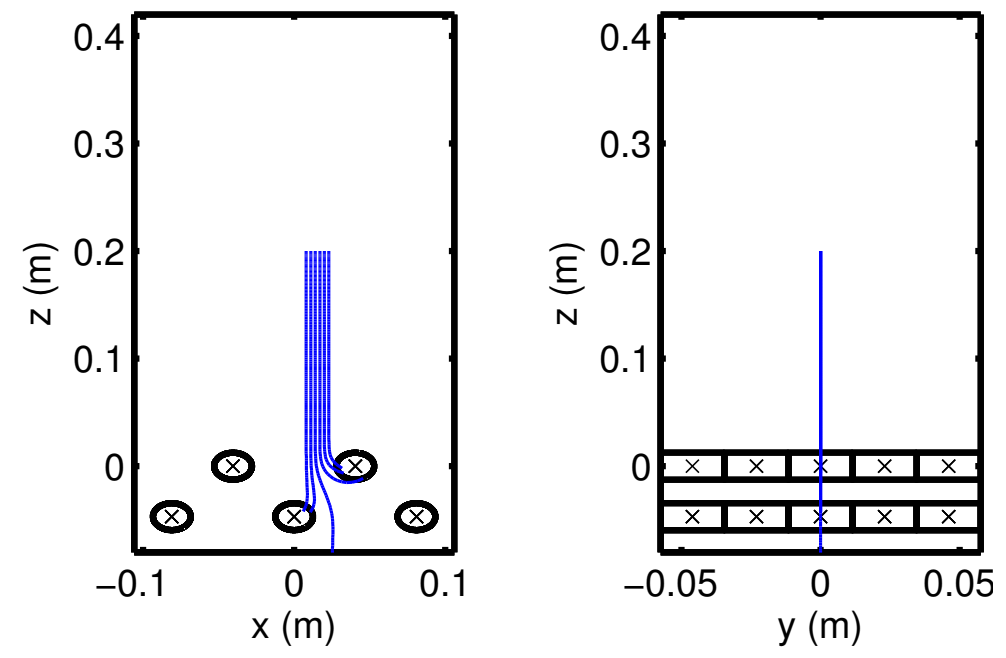

Figure 7: The two rectangular release zones for Monte Carlo simulations
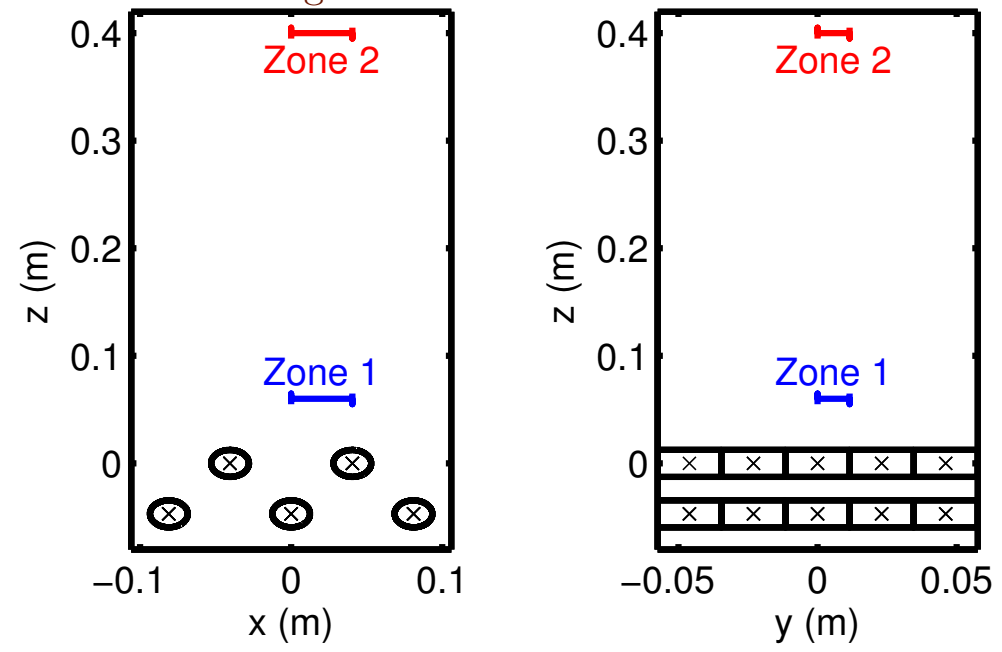
Particles released from Zone 1 had a 97.5\% capture rate whereas Zone 2 had a $94.1 \%$ capture rate. These results have a standard error of $0.5 \%$ : this tolerance was achieved with 1000 simulations from Zone 1 and 2000 from Zone 2.

\section{Discussion}

The simulation model developed above provides a useful tool to determine the proficiency of magnetic particle capture. Monte Carlo simulations have shown that the magnets capture the majority of the magnetised particles.

The Monte Carlo simulations have also shown that significantly more particles are captured when the particles are released from a lower height. This means that the velocity of the particles, as they approach the magnets, is an important factor and should be minimised, where possible, to increase the capture rate.

The simulated particle trajectories in Figures 4-6 show that there are particular paths where the particles pass through the magnet array. These paths tend to go through regions where the magnetic field is weakest such as where there is destructive interference of the magnetic field. There is potential for other vastly different magnetic array orientations to be designed that minimise destructive interference: this has not been investigated in this study.

For particles wider than $10^{-4} \mathrm{~m}$, the size and shape of a particle only has a very small effect on the probability that it is captured by the magnets. This is because, at these widths, the drag is negligible and the drag term in the acceleration equation is the only term that is explicitly affected by the size and shape of the particle. The drag forces become more important for particles that have an effective width less than $10^{-4} \mathrm{~m}$. This is likely to result in a reduction of the probability of capture.

In the Monte Carlo simulations the magnets captured $\sim 95 \%$ of all particles. 
Thus a second set of magnets, placed a distance below the first, would ensure the capture of $\sim 99.75 \%$ of all magnetic particles. This is based on the assumption that the particles are sufficiently randomised after going through the first set of magnets. In addition to the increased capture of particles, a second set of magnets could also be used to monitor the capture rate of the first set of magnets.

\section{Future steps}

In order to accurately determine the capture rate of magnetic particles the model would first need to be experimentally validated and improved as necessary (such as including the full fluid problem for small particles). Estimates of the joint distributions of the particle size, magnetic susceptibilities and particle velocities as they near the magnetic filter would also be needed. After these steps have been carried out, Monte Carlo simulations utilising geometries of individual factories can be completed to accurately determine the capture rate of particles.

\section{References}

[1] Happel, J. (1959) Viscous flow relative to arrays of cylinders. AIChE J., 5: 174177. doi:10.1002/aic.690050211 M335, M339

\section{Author addresses}

\section{H. Cooper,}

mailto:haydn.m.cooper@gmail.com orcid:0000-0001-5325-9436 
2. J. Denier, Macquarie University orcid:0000-0002-5428-2387

3. A. Ali, Auckland University of Technology orcid:0000-0002-5675-9580

4. V. Chopovda, Massey University orcid:0000-0002-2280-5251

5. A. Gulley, The University of Auckland orcid:0000-0003-0759-1897 